\title{
CORRIGENDUM
}

\section{The diversity and host interactions of Propionibacterium acnes bacteriophages on human skin}

Jared Liu, Riceley Yan, Qiao Zhong, Sam Ngo, Nathanael J Bangayan, Lin Nguyen, Timothy Lui, Minghsun Liu, Marie C Erfe, Noah Craft, Shuta Tomida and Huiying Li

The ISME Journal (2015) 9, 2116; doi:10.1038/ismej.2015.144

Correction to: The ISME Journal (2015) 9, 2092-2107; doi:10.1038/ismej.2015.47; published online 7 April 2015

Since the publication of this article, the authors have noticed errors in Figures $4 \mathrm{a}$ and 6 , where one Propionibacterium acnes strain, HL103PA1, was mislabeled in Figure 4a, and the CRISPR spacers were shown incorrectly in Figure 6. The sentences concerning the errors in the article have been corrected accordingly.

The errors have now been rectified in the paper. The authors would like to apologize for any inconvenience this may have caused. 